\title{
Éditorial
}

\section{Événements extrêmes : retours d'expérience}

La multiplication d'événements catastrophiques a marqué les esprits en fin de $X X^{e}$ comme en début de $\mathrm{XXI}^{\mathrm{e}}$ siècle. Certains de ces événements sont qualifiés de naturels : le séisme d'octobre 2005 au Cachemire, le cyclone Katrina sur lequel nous reviendrons, le tsunami de décembre 2004, la canicule de l'été 2003, les tempêtes de 1999, les crues et les inondations dont celles du delta du Gange, les éruptions volcaniques... D'autres sont directement liés aux activités humaines et amènent à douter de la notion même de progrès scientifique : Bhopal, Seveso, Tchernobyl, la «catastrophe de Toulouse » en 2001, les marées noires répétées de l'Alaska et de la Bretagne, sans oublier bien sûr l'usage d'armes de plus en plus destructrices.

La montée en puissance de la notion d'«événements extrêmes » dans le milieu scientifique international est à mettre en relation avec la violence et les effets complexes de ces désastres. Car le terme de catastrophes, dont on ne sait plus dire si elles sont naturelles ou sociales tant les phénomènes sont imbriqués dans nos civilisations modernes, ne suffit plus pour désigner ces « risques majeurs » à la fois imaginables et inimaginables du fait de nos réticences à les appréhender, à les affronter dans toutes leurs dimensions.

Depuis la fin de l'été, le cyclone Katrina met sévèrement en question le rôle des responsables politiques dans nos sociétés " avancées ». Mais cette tragédie, dont les médias soulignent plus encore les aspects sociaux que «naturels » («Lost city », Dennis Overbye, The New York Time Saturday, 10 septembre 2005), interpelle aussi les scientifiques. Ces derniers savaient précisément quelles seraient les conséquences funestes d'un cyclone de force 5 sur le Sud des États-Unis, particulièrement dans un pays où la recherche scientifique s'est illustrée sur les thèmes des Natural Hazards et de la justice spatiale. Après l'alerte de l'ouragan George en 1998, les chercheurs avaient redoublé leurs efforts de simulation et dénoncé, sans retenir l'attention, la dégradation des défenses naturelles de la côte, la vulnérabilité des digues du lac Pontchartrain, le risque d'inondation de la Nouvelle-Orléans, voire la «destruction virtuelle» de la ville par un tel cyclone en provenance du sud-ouest (Mike Davis, Le Monde diplomatique, octobre 2005). Observant les conditions de vie et de circulation, ces chercheurs avaient prévu, sans convaincre davantage, l'impossibilité d'évacuer une part importante de la population - les plus pauvres et les plus démunis - et prévenu des conséquences «presque inimaginables » d'un ouragan d'une force supérieure à 3 . Leurs modèles tournaient et leurs prévisions étaient exactes «à $100 \%$ \%, a-t-on dit plus tard... Trop tard.

Sans doute ne suffit-il pas « de savoir pour accepter ce que l'on sait et agir en conséquence », comme l'exprime Jean-Pierre Dupuy dans sa Petite métaphysique des tsunamis (Le Seuil, 2005). Nous devons pourtant nous interroger : pourquoi les spécialistes ont-ils tant de difficultés pour communiquer leurs connaissances aux responsables politiques? Et comment pourraient-ils y parvenir en temps utiles si «[p]révoir, c'est gouverner » (Natures Sciences Sociétés, 1, 1, 1993) ? Ces questions méritent réflexion en ces temps de réchauffement climatique, d'épuisement des ressources de la planète, de malaria et de recrudescence des épidémies. Faire face à ces événements - ceux que nous ne saurons jamais empêcher comme ceux que nous pouvons éviter - suppose de savoir pourquoi et comment ils se produisent, et comment ils peuvent déclencher des désastres en biens et en vies humaines. Suppose aussi de lutter contre les conditions qui mènent à ces désastres, parmi lesquelles figurent la pauvreté, l'ignorance, les développements économiques trop peu soucieux de justice sociale et de viabilité environnementale. Suppose encore d'organiser de façon plus efficace les alertes et les élans de solidarité.

S'impose alors l'obligation d'informer et de préparer, pour reprendre le titre du rapport publié en 2004 par 1'Organisation météorologique mondiale à propos des catastrophes hydrologiques. Et d'abord d'échanger à propos des événements extrêmes : même rares, ils marquent durablement les systèmes écologiques et sociaux, orientent leurs trajectoires, se superposent à des tendances de fond ressenties sur le long terme. D'échanger sur l'histoire de 
ces événements pour une prise de conscience qui, loin d'être paralysante, soit source de renouveau. Les scientifiques doivent apprendre à tirer les leçons du passé, à établir des hiérarchies dans les modes d'intervention : pour eux-mêmes et pour leurs concitoyens. Pour eux-mêmes, car il s'agit d'analyser les faits et leurs conséquences, d'en dégager les perspectives théoriques et les modalités pratiques. Pour leurs concitoyens, en vertu d'un devoir de partage des connaissances, de solidarité.

Natures Sciences Sociétés a plusieurs fois évoqué ces questions, particulièrement à propos de la notion de risque, en insistant par exemple sur l'intérêt des retours d'expérience et d'une expertise ex post en vue d'une expertise ex ante (Éditorial, 7, 3, 1999). Ces retours d'expérience sont en effet essentiels pour comprendre les réactions des systèmes écologiques et sociaux aux événements extrêmes. $Q u^{\prime}$ en attendre? $Q u^{\prime}$ en tirer pour développer les capacités de résilience de ces systèmes? Comment susciter les solidarités indispensables au niveau des territoires impliqués? Et comment faire prendre conscience des enjeux planétaires? Nous engageons une réflexion en ce sens dès ce numéro, à propos des maladies infectieuses (l'article de J.A. Whittum-Hudson et A.P. Hudson), du rôle des scientifiques dans l'interprétation des risques industriels (le forum de J.-M. Arnaudiès), de la gestion forestière (l'entretien avec M. Badré), des conséquences de l'introduction d'une espèce rentable et «invasive " (la libre opinion de G. Almarcha), des risques naturels et technologiques (textes de vie scientifique de S. Cartier et de D. Terrasson), des textes qu'enrichissent plusieurs comptes rendus d'ouvrages sur Tchernobyl, les tempêtes de 1999, les risques liés à la vache folle et la gestion des déchets. Et la question de l'alimentation n'est pas sans rapport avec cette réflexion (textes du dossier figurant en articles et en forums). Surtout, pour les numéros à venir, nous appelons d'autres témoignages, d'autres analyses d'événements extrêmes, car nous sommes loin d'en mesurer toute la diversité, toute la complexité, toutes les surprises possibles.

Henri Décamps Nicole Mathieu

To access this journal online: www.edpsciences.org 